\title{
Recombinant Human Insulin-Like Growth Factor-1 Treatment: Prime Time or Timeout? [Commentary on "Recombinant Human Insulin Like Growth Factor-1 Treatment: Ready for Prime Time" by Bright GM, Mendoza JR, Rosenfeld RG, Endocrinol Metab Clin N Am 2009; 38:625-38]
}

\author{
Arlan L. Rosenbloom \\ Division of Endocrinology, Department of Pediatrics, College of Medicine, University of Florida, 1701 SW 16th Avenue, \\ Gainesville, FL 32608, USA \\ Correspondence should be addressed to Arlan L. Rosenbloom, rosenal@peds.ufl.edu
}

Received 21 October 2009; Accepted 27 October 2009

Copyright () 2009 Arlan L. Rosenbloom. This is an open access article distributed under the Creative Commons Attribution License, which permits unrestricted use, distribution, and reproduction in any medium, provided the original work is properly cited.

The paper by Bright et al. is an effort to authenticate the recently introduced designation of "primary insulin-like growth factor-1 deficiency (IGFD)" and it's treatment with recombinant human insulin-like growth factor-1 (rhIGF-1).

The concept of "primary IGFD" is supported by comparison to states of hormone deficiency that occur despite adequate stimulation to the target gland, such as primary hypothyroidism and primary hypocortisolism. This is a specious argument. These designations of primary abnormality are not diagnoses but provisional diagnostic categories. While primary hypothyroidism is used as a broad categorization, diagnosis requires, at the very least, differentiation between congenital and acquired, both of which include a wide range of specific diagnoses which have prognostic and therapeutic importance. Similarly, we rarely, if ever, speak of primary hypocortisolism, because of the very limited information or therapeutic guidance conveyed by a categorization that encompasses various forms of congenital adrenal hyperplasia, autoimmune and infectious Addison disease, adrenal leukodystrophy, and congenital adrenal unresponsiveness.

These authors, however, are suggesting that "primary IGFD" is a viable specific diagnosis applicable to a substantial proportion of children with idiopathic short stature (ISS).
Were this proposal validated, the market for recombinant insulin-like growth factor-1 (rhIGF-1) would be greatly expanded beyond the rare conditions resulting in growth hormone $(\mathrm{GH})$ insensitivity for which rhIGF-1 was approved by the US Food and Drug Administration (FDA) [1].

It makes no more sense to refer to $\mathrm{GH}$ insensitivity or resistance as "primary IGFD" than it would to refer to GH deficiency (GHD) as "secondary IGFD" [2]. The problem with this designation as a useful diagnostic entity is seen in the vast differences among the conditions subsumed, from GH receptor and IGF-1 gene mutations, and including acid labile subunit (ALS) mutation as one of the molecular defects listed in Box 1 as causing "primary IGFD." Because ALS is needed to stabilize the complex of IGF-1 and IGFBP3 in the circulation, its absence results in extremely low circulating IGF-1 and IGFBP3 levels. However, there is no impairment of IGF-1 synthesis in any tissue, making the assignment of ALS mutation as a cause of "primary IGFD" irrational. That the ALS deficiency has no effect on paracrine/autocrine IGF1 synthesis or effect is the likely explanation for no-to-modest effect on growth in individuals with ALS deficiency $[3,4]$.

The notion of "primary IGFD" is further advanced with a modification of the Venn diagram that Cohen [5] has used, indicating partial overlap of IGFD and GHD, and of short 
stature and GHD, with a small shaded region overlapping IGFD and short stature. All GHD is characterized by IGFD, however, not simply the $\sim 20 \%$ showing overlap. Similarly, the overlap between short stature and GHD should be nearly complete, excepting only recent onset GHD, or the rare growth without $\mathrm{GH}$ phenomenon. The overlap between IGFD and short stature attributed to "primary IGFD" is hypothetical, with the exception of the small number of proven GH resistant patients who have received rhIGF-1 over the past nearly 20 years ( $\sim 150$ individuals worldwide), which would make the area of overlap miniscule.

In discussing the possible prevalence of "primary IGFD," it is assumed that the prepubertal children recruited into the sponsored clinical trials of rhIGF-1 fit this designation and "may have been categorized in previous clinical trials as having ISS, constitutional delay, or familial short stature." Most of these subjects are, indeed, likely to have constitutional delay in growth and maturation (CDGM), the most common explanation for short stature seen in the pediatric endocrinology clinic [6] and frequently associated with low IGF-1, especially if the IGF-1 levels are interpreted for chronologic age rather than for the delayed bone age or pubertal stage.

The authors go on to note that $17 \%$ of Genentech National Corporative Growth Study (NCGS) subjects were classified as ISS with normal GH responses and no other known etiology. This datum has no apparent relevance for validating "primary IGFD," as emphasized by the statement that it is unknown how many had low serum IGF-1 levels. There is, however, evidence that NCGS subjects without GHD and having low IGF-I not only responded as well to rhGH as those children with normal circulating levels of IGFI, but that their responses correlated inversely with baseline IGF-I concentrations [7-9]. This observation is the opposite of what one would expect if the low IGF-I concentrations were the result of GH insensitivity.

Another line of purported evidence for the concept of "primary IGFD" is that $19 \%-25 \%$ of subjects with short stature and normal GH levels have IGF-1 concentrations below normal. The references fail to support this assertion $[10,11]$. One reference is to a study in which 190 children of 1 month to 20 years of age referred for short stature underwent $\mathrm{GH}$ stimulation testing with arginine. Normal peak GH levels were seen in only $46 \%$ (as would be expected in a largely prepubertal population in the absence of sex steroid priming [12]), of whom $24 \%$ had IGF- $1<-2$ SD, based on a single IGF-1 measurement. There is no information about age distribution of the population within this broad age span, nor stratification according to bone age discrepancy from chronologic age. The younger extreme of the age range would have highly unreliable IGF-1 data and the older range is likely to include many, if not most, with CDGM. IGF-1 SDS was determined by chronological rather than developmental age, which would be inappropriate for those with CDGM. There are no data on response to GH treatment, the obvious gold standard for $\mathrm{GH}$ resistance. Finally, the peak GH concentrations correlated positively with the serum concentrations of IGF-1 only for those with peak GH less than $7.6 \mathrm{mcg} / \mathrm{L}$, accounting for only $9 \%$ of the variation in IGF-1 [10]. Were GH resistance an explanation for the low circulating IGF-1 levels, increasing GH levels would have correlated inversely with IGF-1 concentrations. The other study cited was an examination of the value of IGF-1 measurement in the diagnosis of GHD and concluded, "Our findings cannot fully resolve the controversy regarding the extent to which levels of IGF peptides are important to the diagnostic workup in children with short stature" [11].

While there is recognition of the uncertainty of IGF1 measurement, absent is consideration of the difficult-tocounter argument that children with CDGM should have their IGF-1 levels interpreted for developmental (bone) age rather than chronologic age. The data regarding lower circulating IGF-I concentrations in ISS are not only highly suspect because of the unreliability of the assays, but the populations studied would be expected to include a majority of CDGM who have substantially delayed bone age. Normal values for IGF-1 concentrations are a function of developmental status represented by bone age or Tanner stage. If the IGF1 concentrations are interpreted for chronologic age $[7,10]$, they will typically be lower than normal if there is bone age or pubertal delay for chronologic age. A delayed 14-year-old boy with a bone age of 11 years (a typical presentation) would not have a testosterone level for a typical 14-year-old boy. Similar logic should apply to interpreting IGF-1 measurement, which is also developmental level dependent. In this example, a reading at -2 SDS for chronologic age would be read as -1 SDS for developmental age [13]. Even without this unacknowledged variable, the extensive discussion of the problems with IGF-1 assays and their interpretation does not suggest confidence in a diagnostic criterion based on a single IGF-1 measurement [14].

The authors further advance the notion of varying degrees of GH insensitivity with a novel perspective on a paper coauthored by Bright a quarter of a century ago [15]. One would not have realized that "the concept of an IGF-1 deficiency continuum gained initial attention in the early 1980s" by reading the original paper. Nor would one appreciate without going to the source that "Studies of children who had marked short stature, IGF-1 deficiency, and normal-to-elevated GH levels found that the heights and IGF-1 levels of the subjects were not reduced as severely as for children who had Laron syndrome." refers to a case report of only 2 patients, or that the current description of these patients is revisionary. The range of heights in Laron syndrome encompasses that of the 2 subjects $(-4.8$ $\&-4.0$ height SDS), and the growth velocities were 3.4 and $4.3 \mathrm{~cm} /$ year, also well within the Laron syndrome mean range of 3.0 to $5.0 \mathrm{~cm} /$ year, as noted in Box 1 . To determine whether "... the IGF-1 levels were not reduced as severely as for children who had Laron syndrome," it is necessary to look at data from the same era, using the same units for somatomedin activity. The basal range for the 2 patients of $0.22-0.34 \mathrm{U} / \mathrm{mL}$ is precisely midrange for Laron's patients who had unmistakable GH receptor deficiency, as reported in 1984 [16].

Bright and associates continue this singular interpretation by stating, "These subjects were also capable of limited growth response to pituitary-derived $\mathrm{GH}$, suggesting that 
they may have partial, but not complete, GH insensitivity." In the 1983 paper, however, one patient is reported to have grown at a velocity of $8 \mathrm{~cm} /$ year and the other at $12 \mathrm{~cm} /$ year during the 6-month study period, which in 1983 they described as an excellent response. This interpretation was correct in the context of the era, as a response to pituitary extract GH given 3 times a week instead of the contemporary more effective daily injection, and at a total dose per week that was approximately half the current minimum starting dose of rhGH for GHD. Even at this low dose, somatomedin $\mathrm{C}$ levels increased 3-4 fold. It is disingenuous to state that what was called an excellent response 25 years ago and can still be considered as such, both in terms of growth acceleration and IGF-1 generation, was a "limited response." To suggest that this experience played a role in advancing the notion of a continuum of IGF-1 deficiency related to varying $\mathrm{GH}$ responsiveness is contradicted by the data, and, quite appropriately, was not suggested in the original paper.

It is difficult to see how the discussion of variable effects of various mutations and polymorphisms of the GH receptor argues for "prime time" readiness of rhIGF-1 treatment for other than the approved orphan indications. The closing sentence of the paragraph states, "In 82 subjects who had GH insensitivity syndrome, 16 gene defects were found among 27 subjects; there was no relationship between the site or type of mutation and neither height SDS or biochemical parameters." These were all subjects with Laron syndrome due to GH resistance. There are no genetic data to support the hypothesis of GH insensitivity in the 500 patients recruited by pediatric endocrinologists to the rhIGF-1 trial.

The discussion of experience with rhIGF-1 in Laron syndrome is noteworthy for failing to address the inferior results in comparison to rhGH-treated GH deficiency [17].

The long-term study, on which FDA approval was based, is referenced under the heading "Recombinant human insulin-like growth factor-1 treatment of severe primary insulin-like growth factor I deficiency." This rubric assumes that the subjects in the study with GH gene deletion who developed GH neutralizing antibodies to rhGH (12\% of the group) have "severe primary IGFD." However, this etiology is not listed in the authors' Box 1 "Molecular defects resulting in primary insulin-like growth factor-1 deficiency or insulinlike growth factor-1 resistance (confirmed by molecular analysis)" because they obviously do not have "primary IGFD." The authors of the referenced report avoided taxonomic contortions by accurately referring to their population categorically as having "severe IGF-1 deficiency" which was diagnostically "due to GH insensitivity" [18].

It is stated that studies of children with less severe forms of "primary IGFD" have been going on since 2000, but none are referenced. Instead, a 2007 study of rhGH-treated GHD and what is now being called "primary IGFD" is said to have indicated that the group with "primary IGFD" had less growth despite higher rhGH dosing [19]. However, in the study cited there was no separate "primary IGFD" group, as all of the subjects had low IGF-1 levels. There were 3 study groups, one receiving titrated rhGH doses to achieve an IGF1 SDS of -0.5 to +0.5 , another to achieve an IGF-1 SDS of +1.5 to +2.5 , and the 3rd group to receive a standard
rhGH dose. The first and last groups had nearly identical change in height SDS from baseline over 2 years while the high dose group, not surprisingly, had approximately $50 \%$ greater increase. A majority of these patients (62\%) had normal GH responses to stimulation testing, which may be an underestimate if a substantial number had CDGM and were not primed with a sex steroid for their testing [12]. The responses to rhGH in this study showed that a low IGF-1 with normal GH is not associated with GH insensitivity.

Further support for the view that the "primary IGFD" clinical study subjects did not have GH insensitivity comes from the results of the IGF-1 generation test. The study recruits had greater IGF-1 and IGFBP3 responses to GH than did normals, when the results are expressed as percentage response. These patients had a $76.5 \%$ increase in mean IGF1 level and 34\% increase in mean IGFBP3 concentration in response to 5 days of rhGH administration [20]. Both of these responses are approximately $50 \%$ greater than in normals and confirm GH sensitivity.

Discussion of the two rhIGF-1 clinical trials provides further evidence for the null hypothesis regarding "primary IGFD." The mean growth velocity of the control group, as is typical of CDGM and familial short stature, was normal, which indicates normal GH-IGF-1 activity. Therapy with twice daily rhIGF-1 significantly increased height SDS, as would also be expected with rhGH treatment, for which there was not an arm. Noteworthy is that some subjects had even better first-year height velocities with once daily dosing, which has been explained appropriately as lack of suppression of GH for half of the day, another strong indication of normal GH sensitivity [21].

Review of the safety of rhIGF-1 treatment begins with a statement that, "Naturally occurring substances such as insulin, GH, and IGF-1 that are developed as medications using recombinant DNA technologies have an advantage over other types of medication, because they are integral components of normal physiologic processes. Thus, they have clear mechanisms of action, and the consequences of their deficiency or excess are defined well." It is difficult to imagine why these peptides would be considered more efficacious or safer and their effects more clearly defined than manufactured hormones, such as cortisol, thyroid, testosterone, or estrogen, which are also integral components of normal physiologic processes and no more or less likely to cause trouble when provided in pharmacologic rather than physiologic doses.

For a product to be ready for "prime time," safety and efficacy must be reasonably assured. When the condition to be treated does not threaten health or life, safety is the paramount consideration. Two abstracts are cited by Bright et al. to suggest that "primary IGFD" patients have fewer adverse events than has been documented in longer-term treatment of GH insensitivity $[22,23]$. Neither of these abstracts is retrievable. There is no explanation why children with ISS might be less likely to develop adverse events from rhIGF-1 than patients with true GH resistance.

Considerable information is available from rhIGF-1 treatment for the approved indications, which fails to support the statement that the adverse event frequency 
for rhIGF-1 is lower for children with "primary IGFD" than for those with true GH insensitivity, or that the frequency is consistent with that for rhGH treatment. Adverse events for patients with GH insensitivity treated with rhIGF-1 are cumulative, making it inappropriate to compare short-term studies to these longer-term studies without correcting for patient years. For example, over $10 \%$ of subjects will require tonsillectomy/adenoidectomy as a result of the lymphoid hyperplasia that develops in $25 \%$ of patients and is associated with hypoacusis and snoring, which will increase from $4 \%$ in the 1 st year to $65 \%$ after several years of treatment [18]. Tympanostomy tubes will be required in 16\%. Nephrolithiasis has developed in $3 \%$ of patients. Acromegaloid coarsening of the facial features with disproportionate growth of the jaw is common, particularly in adolescent subjects. Disproportionate fat mass increase is typical and severe obesity has occasionally occurred. Parotid swelling and facial nerve palsy have been described. None of these are adverse effects of treatment with rhGH.

Benign intracranial hypertension (BIH) has been seen in $4 \%$ of patients with GH receptor deficiency or GH inactivating antibodies treated with rhIGF-1, for an estimated 90/10,000 patient years [18]; recent data from the Tercica clinical trial provide a similar rate of $140 / 10,000$ patient years for those receiving therapeutic doses [24]. The rate for $\mathrm{BIH}$ in rhGH-treated GHD in the NCGS is comparatively small, 15/10,000 patient years [25]. The difference in rates of BIH with rhIGF-1 from rhGH-treated ISS may be the more appropriate comparison. Among over 5000 rhGH-treated subjects with ISS in the Pfizer International Growth Study database and 4500 in the NCGS, there were no instances of $\mathrm{BIH}$ in a combined 25,000 patient years of treatment $[25,26]$. For headache, there is a $>100$ fold difference, $28 / 10,000$ patient years ( $\mathrm{rhGH}$ ) versus $3460 / 10,000$ patient years (rhIGF-1) in the long-term study of severe GH insensitivity and a remarkably similar 3000/10,000 patient years from the recent clinical trial in "primary IGFD" [18, $24,26]$. There were 10 arthralgia or myalgia events per 10,000 rhGH treatment years; the frequency with rhIGF-1 treatment of GH insensitivity is over 600/10,000 treatment years $[18,27]$.

There is also recent concern about potentially lifethreatening allergic reactions, with reports of anaphylaxis and generalized urticaria $[28,29]$. Considering the relatively small number of children who have been treated, two reports of serious allergic reactions associated with rhIGF-1 treatment indicate the need to add major and minor allergic reactions to the list of adverse events associated with rhIGF-1 treatment.

In the section on dose selection, it is stated that the goal was to increase serum IGF-1 concentrations to the upper end of the normal range for age, that is, +2 SDS. This is a confirmation of the need to attain pharmacologic concentrations, rather than the physiologic (replacement) levels which would be the case with genuine hormonal deficiency states, such as hypothyroidism and adrenal insufficiency.

After declaring that dose selection was based on the goal of attaining circulating IGF- 1 levels of +2 SDS, the statement is made that outcomes are not linked to serum IGF-1 levels during treatment with rhIGF-1. These 2 statements appear dissonant. In fact, the 2-year treatment study of Ecuadorian subjects with GH receptor deficiency showed significant association between IGF-1 levels attained and growth response. For the 1st year of treatment, change in height age correlated with trough levels of IGF-1 with a $P$ value of 0.009 , and in the 2nd year of 0.005 [17].

The authors state that it has become increasingly clear that the major molecular defects described thus far to explain "primary IGFD" represent only one end of the IGFD spectrum and repeat the unsupportable 19\% and 25\% figures discussed earlier. They go on to claim that the European and US approvals of rhIGF-1 for severe primary IGFD support the concept of milder "primary IGFD," which is a huge speculative leap. Having made that leap, the authors indicate that it is apparent that a sizable subset of ISS should no longer be classified as such, but as "primary IGFD" and by this reasoning whether rhIGF-1 should be optimal therapy. As noted above, evidence for GH insensitivity in this group remains to be found.

The compilation of evidence to challenge the assumption of "primary IGFD" as part of the spectrum of GH resistance explaining a substantial portion of ISS is completed when the authors present an argument for combination rhGH and rhIGF-1 therapy which, by definition, requires normal GH sensitivity to be effective.

In the discussion of combination therapy, reference is once again made to the study of a group of children with low IGF-1 levels of whom $\sim 2 / 3$ had normal GH responses as if they had been studied in 2 groups, GH deficiency and primary IGFD, but they were not [19]. They emphasize the need for achieving high concentrations of IGF-1 with rhGH for therapeutic effect; this requirement would be inconsistent with GH insensitivity or "primary IGFD." Rather, these observations reflect the absence of a hormonal etiology, requiring pharmacologic rather than physiologic levels of IGF-1 for growth promotion. There is also an absence of consideration that $\mathrm{GH}$ administration does not simply increase endocrine IGF-1 concentration. There is, however, appreciation of the fact that rhIGF-1 monotherapy causes suppression of nocturnal GH secretion. This recognition and the suggestion that combination therapy might outperform either rhGH or rhIGF-1 monotherapies counters the argument that GH insensitivity is the core problem for which rhIGF-1 is indicated.

The basis for the promotion of rhIGF-1 reflected in the paper of Bright et al. has been noted in the interesting and informative book Normal at Any Cost [30]:

"In a replay of Genentech's strategy with growth hormone decades earlier, Tercica received orphan drug status based on the rarity of the disorder, then immediately set out to expand the market.... As Tercica's president and CEO celebrated [FDA] approval, he described to the business press how sales reps and managers would be dispatched to talk to pediatric endocrinologists "who form the basis of this short stature market... building a franchise in short stature and associated metabolic disease." Again, echoes of the recombinant growth hormone story.... Tercica estimated some 60,000 children evenly 
split between the United States and Western Europe have short stature with some lesser degree of growth hormone insensitivity, representing a $\$ 1$ billion annual market opportunity. The company would conduct continuing medical education programs, medical symposia, and regional speaker programs "aimed at establishing awareness of Increlex in the physician community"."

\section{Acknowledgment}

Conflict of interest statement: A. L. Rosenbloom has received a consulting honorarium and travel expenses from Veraxis for a presentation regarding "IGFD" and travel expense coverage for a meeting of the Genentech National Cooperative Growth Study, both in 2009.

\section{References}

[1] A. L. Rosenbloom, "Is there a role for recombinant insulin-like growth factor-I in the treatment of idiopathic short stature?" The Lancet, vol. 368, no. 9535, pp. 612-616, 2006.

[2] S. Rosenthal, "Part I: clinical diagnosis of IGF-I deficiency," Pediatric Endocrinology Reviews, vol. 4, supplement 2, pp. 252 256, 2007.

[3] A. A. Butler and D. Le Roith, "Control of growth by the somatropic axis: growth hormone and the insulin-like growth factors have related and independent roles," Annual Review of Physiology, vol. 63, pp. 141-164, 2001.

[4] O. V. Fofanova-Gambetti, V. Hwa, S. Kirsch, et al., "Three novel IGFALS gene mutations resulting in total ALS and severe circulating IGF-I/IGFBP-3 deficiency in children of different ethnic origins," Hormone Research, vol. 71, no. 2, pp. 100-110, 2009.

[5] P. Cohen, "Problems with reclassification of insulin-like growth factor I production and action disorders," Journal of Clinical Endocrinology and Metabolism, vol. 91, no. 11, pp. 4235-4236, 2006.

[6] A. Grimberg and F. Lifshitz, "Worrisome growth," in Pediatric Endocrinology, F. Lifshitz, Ed., vol. 2, pp. 1-50, Marcel Dekker, New York, NY, USA, 5th edition, 2007.

[7] K. M. Attie, L. M. S. Carlsson, A. C. Rundle, and B. M. Sherman, "Evidence for partial growth hormone insensitivity among patients with idiopathic short stature," Journal of Pediatrics, vol. 127, no. 2, pp. 244-250, 1995.

[8] P. Park and P. Cohen, "Insulin-like growth factor I (IGF-I) measurements in growth hormone $(\mathrm{GH})$ therapy of idiopathic short stature (ISS)," Growth Hormone and IGF Research, vol. 15, supplement 1, pp. S13-S20, 2005.

[9] M. B. Ranke, R. Schweizer, M. W. Elmlinger, et al., "Relevance of IGF-I, IGFBP-3, and IGFBP-2 measurements during GH treatment of $\mathrm{GH}$-deficient and non-GH-deficient children and adolescents," Hormone Research, vol. 55, no. 3, pp. 115124, 2001.

[10] P. E. Clayton, O. Ayoola, and A. J. Whatmore, "Patient selection for IGF-I therapy," Hormone Research, vol. 65, supplement 1, pp. 28-34, 2006.

[11] M. B. Ranke, R. Schweizer, M. W. Elmlinger, et al., "Significance of basal IGF-I, IGFBP-3 and IGFBP-2 measurements in the diagnostics of short stature in children," Hormone Research, vol. 54, no. 2, pp. 60-68, 2000.

[12] G. Marin, H. M. Domené, K. M. Barnes, B. J. Blackwell, F. G. Cassorla, and G. B. Cutler Jr., "The effects of estrogen priming and puberty on the growth hormone response to standardized treadmill exercise and arginine-insulin in normal girls and boys," Journal of Clinical Endocrinology and Metabolism, vol. 79, no. 2, pp. 537-541, 1994.

[13] A. L. Rosenbloom, "Idiopathic short stature: conundrums of definition and treatment," International Journal of Pediatric Endocrinology, vol. 2009, Article ID 470378, 5 pages, 2009.

[14] J. Khosravi, A. Diamandi, U. Bodani, N. Khaja, and R. G. Krishna, "Pitfalls of immunoassay and sample for IGF-I: comparison of different assay methodologies using various fresh and stored serum samples," Clinical Biochemistry, vol. 38, no. 7, pp. 659-666, 2005.

[15] G. M. Bright, A. D. Rogol, A. J. Johanson, and R. M. Blizzard, "Short stature associated with normal growth hormone and decreased somatomedin-C concentrations: response to exogenous growth hormone," Pediatrics, vol. 71, no. 4, pp. 576-580, 1983.

[16] Z. Laron, "Laron-type dwarfism (hereditary somatomedin deficiency): a review," Ergebnisse der Inneren Medizin und Kinderheilkunde, vol. 51, pp. 117-150, 1984.

[17] J. Guevara-Aguirre, A. L. Rosenbloom, O. Vasconez, et al., "Two-year treatment of growth hormone $(\mathrm{GH})$ receptor deficiency with recombinant insulin-like growth factor I in 22 children: comparison of two dosage levels and to GHtreated GH deficiency," Journal of Clinical Endocrinology and Metabolism, vol. 82, no. 2, pp. 629-633, 1997.

[18] S. D. Chernausek, P. F. Backeljauw, J. Frane, J. Kuntze, and L. E. Underwood, "Long-term treatment with recombinant insulin-like growth factor (IGF)-I in children with severe IGFI deficiency due to growth hormone insensitivity," Journal of Clinical Endocrinology and Metabolism, vol. 92, no. 3, pp. 902910, 2007.

[19] P. Cohen, A. D. Rogol, C. P. Howard, G. M. Bright, A.-M. Kappelgaard, and R. G. Rosenfeld, "Insulin growth factorbased dosing of growth hormone therapy in children: a randomized, controlled study," Journal of Clinical Endocrinology and Metabolism, vol. 92, no. 7, pp. 2480-2486, 2007.

[20] G. M. Bright, C. K. Buckway, J. W. Frane, and R. G. Rosenfeld, "IGF-1 generation test results in prepubertal children with primary IGF-1 deficiency suggest GH insensitivity," 2009, http://IGFDforum.com.

[21] L. K. Midyett and A. Rogol, "Clinical trial update: firstyear height velocities with once daily and twice-daily dosing of rhIGF-1 in prepubertal children with primary IGF-1 deficiency," 2009, http://IGFDforum.com.

[22] L. K. Midyett, A. Rogol, J. Frane, et al., "Efficacy and safety of twice-daily rhIGF-1 treatment in prepubertal children with Primary IGF-1 deficiency: results from a randomized clinical trial," in Proceedings of the ICE Meeting, Rio de Janeiro, Brazil, November 2008.

[23] G. M. Bright, D. Rogers, L. G. Mendoza, et al., "Safety and efficacy of once-daily rhIGF-1 treatment in prepubertal children with primary IGF-1 deficiency:results from a clinical trial," in Proceedings of the ICE Meeting, Rio de Janeiro, Brazil, November 2008.

[24] L. K. Midyett, D. Rogers, D. Hale, A. D. Rogol, J. W. Frane, and G. M. Bright, "First-year height velocities with once-daily or twice-daily dosing in two clinical trials of prepubertal children with primary IGF-I deficiency," in Proceedings of the Annual Meeting of The Endocrine Society, Washington, DC, USA, June 2009, Poster \#P1-742.

[25] S. L. Blethen, D. B. Allen, D. Graves, G. August, T. Moshang, and R. Rosenfeld, "Safety of recombinant deoxyribonucleic acid-derived growth hormone: the national cooperative 
growth study experience," Journal of Clinical Endocrinology and Metabolism, vol. 81, no. 5, pp. 1704-1710, 1996.

[26] F. Darendeliler, G. Karagiannis, and P. Wilton, "Headache, idiopathic intracranial hypertension and slipped capital femoral epiphysis during growth hormone treatment: a safety update from the KIGS database," Hormone Research, vol. 68, supplement 5, pp. 41-47, 2007.

[27] P. Wilton, "Adverse events during GH treatment: 10 years' experience in KIGS, a pharmacoepidemiologic survey," in Growth Hormone Therapy in KIGS-10 Years' Experience, M. B. Ranke and P. Wilton, Eds., pp. 349-364, Johann Ambrosius Barth, Heidelberg, Germany, 1999.

[28] E. Torjusen, J. Calderon, and S. A. Rivkees, "Anaphylactic reaction to recombinant insulin-like growth factor-I," Journal of Pediatric Endocrinology and Metabolism, vol. 21, no. 4, pp. 381-384, 2008.

[29] K. A. Metz, A. Assa'ad, M. B. Lierl, and P. Backeljauw, "Allergic reaction to mecasermin," Annals of Allergy, Asthma and Immunology, vol. 103, no. 1, pp. 82-83, 2009.

[30] S. Cohen and C. Cosgrove, Normal at Any Cost: Tall Girls, Short Boys, and the Medical Industry's Quest to Manipulate Height, Penguin Group, New York, NY, USA, 2009. 